\title{
Galaxy merging and the Fundamental Plane of elliptical galaxies
}

\author{
Carlo Nipoti ${ }^{1}$, Pasquale Londrillo ${ }^{2}$, and Luca Ciotti ${ }^{2,3}$ \\ 1 Dipartimento di Astronomia dell'Università degli Studi di Bologna, via Ranzani 1, \\ 40127 Bologna, Italy \\ 2 Osservatorio Astronomico di Bologna, via Ranzani 1, 40127 Bologna, Italy \\ 3 Scuola Normale Superiore, Piazza dei Cavalieri 7, 56126 Pisa, Italy
}

\begin{abstract}
We present preliminary results of numerical simulations of dissipationless merging of stellar systems, aimed at exploring the consequences of merging between gas free, spheroidal systems. In particular, we study the dynamical and structural characteristics of hierarchical merging between equal mass stellar systems, and we compare the properties of the end-products with the most important structural and dynamical scaling relations obeyed by spheroids. In the explored hierarchy of four successive mergings we find that the FP tilt is marginally conserved, but both the Faber-Jackson and Kormendy relations are not conserved.
\end{abstract}

\section{Introduction}

From a theoretical point of view, in the scenario of hierarchical galaxy formation elliptical galaxies (Es) formed by merging of smaller systems [4, 11. 12]. On the other hand, from observations we know that Es satisfy many tight scaling relations: for example the Fundamental Plane [5.6] (FP), the $M_{\mathrm{BH}}-\sigma_{0}$ [8. 9], the $\mathrm{Mg}_{2}-\sigma_{0}$ [1], and the color-magnitude [2] relations. In particular, the FP of Es relates their central velocity dispersion $\sigma_{0}^{2}$, total luminosity $L_{\mathrm{B}}$ and circularized effective radius $\langle R\rangle_{\mathrm{e}}$, with a 1-sigma scatter of $\simeq 15 \%$ in $\langle R\rangle_{\mathrm{e}}$ for fixed $L_{\mathrm{B}}$ and $\sigma_{0}$ [10].

Here we focus on the constraints imposed by the existence of the FP on the role of dissipationless merging in the formation of Es. In other words we want to verify, by using numerical simulations, whether the FP is "closed" with respect to the merging process.

Among the motivations of this exploration is the fact that, in the merging of two galaxies with masses $\left(M_{1}, M_{2}\right)$ and virial velocity dispersions $\left(\sigma_{v, 1}, \sigma_{v, 2}\right)$, the virial velocity dispersion of the merger (in case of no mass loss and negligible initial interaction energy of the galaxy pair when compared to their internal energies) is given by

$$
\sigma_{v, 1+2}^{2}=\frac{M_{1} \sigma_{v, 1}^{2}+M_{2} \sigma_{v, 2}^{2}}{M_{1}+M_{2}}
$$

It follows that $\sigma_{v, 1+2} \leq \max \left(\sigma_{v, 1}, \sigma_{v, 2}\right)$, i.e., the virial velocity dispersion cannot increase in a merging process of the kind described above. On the other hand, the Faber-Jackson relation [7] (FJ) indicates that the projected central velocity dispersion increases with galaxy luminosity. In addition, since in a purely gas 
Carlo Nipoti et al.

free merging process the stellar mass-to-light ratio cannot increase, the FP can be maintained only by structural and/or dynamical non-homology.

\section{Results}

The details of the adopted (one and two component) numerical models are given in [14]. We describe here the case of a merging hierarchy: in other words, we merge together the end-products of previous mergers up to four generations, for a total increase of mass of a factor of 16 .

- As expected, in all the merging events $\sigma_{v, 1+2}$ does not differ significantly from those of the progenitors (the largest deviation, due to particle escape, is less than $4 \%$ ).

- On the contrary, the luminosity-weighted projected velocity dispersion inside $\langle R\rangle_{\mathrm{e}} / 8$ of the end-products $\sigma_{a}\left(\langle R\rangle_{\mathrm{e}} / 8\right.$ ) (an estimate of the observed $\sigma_{0}$ ), is larger than in the progenitors, while $\sigma_{a}(\infty) \simeq \sigma_{v, 1+2}$, in accordance with the projected virial theorem [3].

- In general the end-products have $\sigma_{0}$ lower and $\langle R\rangle_{\mathrm{e}}$ larger than those predicted by the FJ and Kormendy [13] relations, respectively. The effects curiously compensate and the end-products remain near the FP: however, the scatter in $\langle R\rangle_{\mathrm{e}}$ can be as large as $35 \%$, when compared to $\langle R\rangle_{\mathrm{e}}$ derived from the FP relation when using the total luminosity (mass) and central velocity dispersion of the end-products.

We are now running high-resolution numerical simulations analogous to those described here, in order to exclude numerical effects on the above results, and to quantitatively check the effects of massive dark matter halos on the properties of the end-products.

\section{References}

1. R. Bender, D. Burstein, S.M. Faber: ApJ, 411, 153 (1993)

2. R.G. Bower, J.R. Lucey, R.S. Ellis: MNRAS, 254, 601 (1992)

3. L. Ciotti: Celest. Mech. \& Dyn. Astron., 60, 401 (1994)

4. S. Cole, C.G. Lacey, C.M. Baugh, C.S. Frenk: MNRAS, 319, 168 (2000)

5. G. Djorgovsky, S. Davis: ApJ, 313, 59 (1987)

6. A. Dressler, S.M. Faber, D. Burstein, R.L. Davies, D. Lynden-Bell, R.J. Terlevich, G. Wegner: ApJ, 313, 37 (1987)

7. S.M. Faber, R.E. Jackson: ApJ, 204, 668 (1976)

8. L. Ferrarese, D. Merritt: ApJ, 539, L9 (2000)

9. K. Gebhardt, et al.: ApJ, 539, L13 (2000)

10. I. Jorgensen, M. Franx, P. Kjaergaard: MNRAS, 280, 167 (1996)

11. G. Kauffmann: MNRAS, 281, 487 (1996)

12. S.D.M. White, M.J. Rees: MNRAS, 183, 341 (1978)

13. J. Kormendy: ApJ, 218, 333 (1977)

14. C. Nipoti, P. Londrillo, L. Ciotti: MNRAS, submitted (2001) 